\title{
Ion cascade acceleration from the interaction of a relativistic femtosecond laser pulse with a narrow thin target
}

\author{
Feng He, ${ }^{a, b}$ Han Xu, ${ }^{b, d}$ Youwei Tian, ${ }^{b}$ Wei Yu, ${ }^{b}$ Peixiang Lu, ${ }^{c}$ and Ruxin $\mathrm{Li}^{b}$ \\ ${ }^{a}$ Max-Planck-Institut für Physik Komplexer Systeme, \\ Nöthnitzer Str.38, D-01187 Dresden, Germany \\ ${ }^{b}$ State Key Laboratory of High Field Physics, \\ Shanghai Institute of Optics and Fine Mechanics, Shanghai 201800, P. R. China \\ ${ }^{c}$ State Key Laboratory of Laser Technology, \\ Huazhong University of Science and Technology, Wuhan 430070, P. R. China \\ ${ }^{d}$ Department of Applied Physics, National University of \\ Deference Technology, Changsha 410073, P. R .China
}

(Dated:)

\begin{abstract}
Particle-in-cell simulations are performed to study the acceleration of ions due to the interaction of a relativistic femtosecond laser pulse with a narrow thin target. The numerical results show that ions can be accelerated in a cascade by two electrostatic fields, if the width of the target is smaller than the laser beam waist. The first field is formed in front of the target by the central part of the laser beam, which pushes the electron layer inwards. The major part of the abaxial laser energy propagates along the edges to the rear side of the target and pulls out some hot electrons from the edges of the target, which form another electrostatic field at the rear side of the target. The ions from the front surface are accelerated stepwise by these two electrostatic fields to high energies at the rear side of the target. The simulations show that the ion energy gain for a narrow target is about four times higher than in the case of a wide target.
\end{abstract}

PACS numbers: $52.38 . \mathrm{Kd}, 52.50 . \mathrm{Jm}, 52.65 . \mathrm{Rr}$ 


\section{INTRODUCTION}

The generation of high energetic ions is very important because of its wide range of applications in therapy and biology [1], fast ignition [2], nuclear physics [3], and others. In several experiments [4-9] and particle-in-cell (PIC) simulations [10-15] it has been shown that ions with $\mathrm{MeV}$ energies can be obtained from the interaction of femtosecond laser pulses with solid targets [16-20], gas jets [21, 22] and clusters [23]. The ions are accelerated by either a Coulomb field via charge separation, a shock wave, or an electrostatic field at the rear side of the target.

One key problem for ion acceleration is to generate strong electrostatic fields. A thin foil target is an ideal medium, since ions, generated by the interaction with the laser beam in the target, can be accelerated both at the front or the rear side of the target. At the front side the laser ponderomotive force pushes the electrons inwards, creating an electric field by charge separation, which drags the ions. Further, some of the hot electrons may exit the target at its rear side and generate a Debye sheath with an electrostatic field of about a MV per micron [17], which will accelerate the ions at the rear side. Recently, Pukhov [11] studied both processes using 3D PIC simulations. A quantitative comparison [24] showed that the acceleration from the rear surface is usually more effective. The field strength at the front side of the target can be increased by using an overdense target, which however in turn decreases the field strength at the rear side, since less electrons penetrate through the high density plasma layer. An alternative mechanism has been discussed by Zhidkov et al. [25], who showed that using a picosecond intense laser pulse the ions can be first accelerated by a shock wave and then further by the electrostatic field at the rear side of the target. In this way the acceleration lasts for several picoseconds.

In this paper we propose another mechanism to obtain high energetic ions. In this scheme a relativistic femtosecond laser pulse irradiates an overdense narrow target, which width is smaller than the laser beam waist. During the interaction the central part of the laser beam is reflected by the target, while most of the abaxial laser energy propagates along the edges to the rear side of the target. The central part of the laser beam pushes the electron layer into the target and a first electrostatic field is formed at the front surface of the target as discussed above. In addition, many electrons at the edges of the target are pulled out by the abaxial laser pulse and pushed towards the rear side of the target, where a second 
electrostatic field is formed. Ions, accelerated at the front surface of the target, enter into this second electrostatic field and are cascade-accelerated to high energies. As it will be shown below the ion energy gain is up to four times larger than in the case of a wide target; e.g. for $I \lambda^{2}=1.37 \times 10^{20} \mathrm{~W} \mu \mathrm{m}^{2} / \mathrm{cm}^{2}$, where $\lambda$ is the wavelength and $I$ is the intensity of the laser pulse, the ion energy gain is found to be about $50 \mathrm{MeV}$. The transverse ponderomotive force at the back side of the target confines the electrons around the axis, which results in a collimated ion beam in this mechanism.

The rest of this paper is organized as follows. In Sec. II, we first introduce the simulation model and the parameters used in the calculations. Then we present the results of the simulations for the interaction of the laser pulse with the narrow target, namely the electron and ion density distributions and the two parts of the electrostatic fields. Next, a comparison between the results obtained for narrow and wide targets are shown in the third part of Sec. II, followed by a general discussion in the last part of Sec. II. Finally, in Sec. III we present the conclusions.

\section{PIC SIMULATIONS}

\section{A. Model and simulation parameters}

Here we present our fully relativistic 2D3V [26] (two dimensions in coordinates and three dimensions in velocities) particle-in-cell simulations for the ion cascade acceleration mechanism. In the 2D3V code the third coordinate dimension is assumed to be periodic. Since the mechanism for ion acceleration is dominated by the longitudinal electrostatic field, the 2D3V code is a good approximation to analyze the ion acceleration along the laser propagation axis. In the calculations we have considered a circularly polarized laser pulse with a central wavelength of $\lambda=1 \mu \mathrm{m}$ and a peak intensity of $I_{0}=1.38 \times 10^{20} \mathrm{~W} / \mathrm{cm}^{2}$, incident on a plasma layer of density $n=5 n_{c}$, where $n_{c}=m \omega^{2} / 4 \pi e^{2}$ is the critical electron density, $m, e, \omega$ are the electron mass, the electron charge and the laser frequency, respectively. The laser pulse profile in time and space is given by $I=I_{0} \exp ^{2}\left(-\rho^{2} / \rho_{0}^{2}\right) \sin ^{2}[\pi(x-c t) / L]$ for $0 \leq x-c t \leq L$. The pulse width is $L=10 \lambda$ and the focal spot radius is $\rho_{0}=5 \lambda$. The overdense plasma target is located at $x=20 \lambda$, its thickness and width are $1 \lambda$ and $3 \lambda$, respectively. The initial plasma density is assumed to be uniform. The full simulation box has 
a size of $x \times y=80 \lambda \times 30 \lambda$ with a grid of $1600 \times 600$ cells, where the $\mathrm{x}$-direction determines the direction of the laser propagation. The transverse boundary conditions are periodic and the longitudinal boundaries are chosen such that the outgoing radiation and particles are absorbed. Both the initial electron and ion temperature are $1 \mathrm{KeV}$. The electric field $E$, time $t$ and the $x$-coordinate are normalized by: $E \rightarrow e E / m \omega v_{t h}, t \rightarrow \omega t, x \rightarrow k x$, where $v_{t h}$ is the initial thermal velocity, and $k$ is the wave vector.

\section{B. Numerical results}

In Fig. 1 we present the transversal field distribution $E_{y}$ at two time instants, namely (a) $\mathrm{t}=33 \mathrm{~T}$ and (b) $\mathrm{t}=41 \mathrm{~T}$, where $T=3.3 \mathrm{fs}$ is the laser period. At $t=33 T$ the laser pulse just touches the target, the target acts like a tiny barrier and causes the laser pulse to diffract. Since the beam waist is much wider than the target, most of the laser energy propagates along the edges of the target. Consequently, the main part of the electromagnetic field is distributed at the back side of the target at $t=41 T$, while the central part of the laser beam is reflected by the target. Thus, at the back side of the target the intensity along the propagation axis is weak. Hence, the radial ponderomotive force points towards the axis, which will cause a confinement of the collimated electron beam near the axis.

Fig. 2 shows snapshots of the electron $[(a),(b)]$ and the ion $[(c),(d)]$ distributions in real space $(\mathrm{x}, \mathrm{y})$ at two time instants $[(\mathrm{a}),(\mathrm{c}) \mathrm{t}=41 \mathrm{~T}$ and $(\mathrm{b}),(\mathrm{d}) \mathrm{t}=58 \mathrm{~T}]$. From Fig.2(a) one can clearly see that many electrons are pulled out by the electric field from the edges of the target and accelerated to high energies at the back side of the target. The energetic electron bunches appear at each cycle of the laser pulse. Due to the charge separation, a strong electrostatic field is generated, which drags the ions. At $t=41 T$, the acceleration of the ions has just started (Fig. 2(c)), while at $t=58 \mathrm{~T}$ a part of the ions appear already at more than $5 \lambda$, showing that they have gained a high energy. The energetic electrons at the back side of the target are confined by the transverse ponderomotive force (Fig. 2b)), which results in a well collimated longitudinal electrostatic field.

In order to get further insight into the mechanism of the ion acceleration, we present in Fig. 3 the longitudinal electrostatic field in the $\mathrm{x}$-axis at $t=25 T$ (dotted line), $37 T$ (solid line) and $49 \mathrm{~T}$ (dashed-dotted line), respectively. The insert represents the enlargement for $x=18-22 \lambda$. At $t=25 T$, the electrostatic field is created at the front surface of the 
target. At this moment, the surface electron layer begins to be pushed into the target by the longitudinal ponderomotive force. The electron density gets larger and the laser energy along the propagation axis is obstructed by the high density plasma. At this time, the laser cannot propagate to the rear side of the target, and there is no electrostatic field. At $t=37 T$, the electrostatic field has two maxima. One peak appears at $x=20.6 \lambda$, and the corresponding electrostatic field is larger than 150. Another peak is located at $x=21.3 \lambda$. Although its peak value is only 50, the line profile is very wide. Thus, the ions can also be accelerated in this area effectively. At $t=49 T$, the former peak is decreased to 30 , while the latter peak is still 50. Additionally, a small fraction of the reflected laser pulse draws back some electrons from the surface of the target and generates a negative electrostatic field, as shown by the dashed-dotted line. From these results, one can conclude that the laser ponderomotive force generates a first electrostatic field, some ions at the front surface of the target will be accelerated quickly by the first electrostatic field and enter the second electrostatic field at the rear side of the target. Because the latter field profile is wide, the ions will be further accelerated continuously. Due to this the ion energy gain is four times larger than in the case of a wide target, as we will show in the next subsection.

\section{Comparison of narrow and wide targets}

For comparison, the ion energy gains obtained by the irradiation of a wide and a small target are shown in Fig. 4 (a) and (b), respectively. For a wide overdense target (Fig. 4 (a)), only the first electrostatic field is formed at the front surface of the target. The mean ion energy can be estimated from the momentum conservation in the case of total back reflection of the center laser beam. We use $n_{i} W_{1}=2 I / c[12,15]$, where $n_{i}$ is the ion density, $c$ is the light velocity, and $I$ is the laser intensity, and estimate the ion energy as $W_{1}=11.4 \mathrm{MeV}$. If the laser pulse irradiates the narrow target, the ions are accelerated by two electrostatic fields and obtain even higher ion energies. From the results in Fig. 4(b) we see that the most energetic ions appear at $x=26.14 \lambda$ and estimate the drift distance in the electrostatic field as $d=5.14 \lambda$. The normalized maximum electrostatic field is about 50 (corresponding to $E_{s}=7.24 \times 10^{12} \mathrm{~V} / \mathrm{m}$ ), and the energy gain from the second electrostatic field is $W_{2}=e E_{s} d=37.2 \mathrm{MeV}$. Thus, the total energy gain for the most energetic ions is $W=W_{1}+W_{2}=48.6 \mathrm{MeV}$. This estimate is in excellent agreement with the simulation 
results.

\section{Discussion}

In our simulations, the normalized vector potential $a=10$ and the corresponding critical plasma density $n_{c}^{\prime}=\sqrt{1+a^{2}} n_{c} \simeq 10 n_{c}$, which is much larger than the target density in the simulations. One may argue that such a target is transparent for such an intense laser pulse, and the whole laser pulse can penetrate through the thin target. However, during the interaction of the laser and the target, the electrons in the front layer of the target are pushed inwards by the ponderomotive force and accumulate inside the target, which creates an instantaneous electron density larger than $n_{c}^{\prime}$. To illustrate this effect we show in Fig.5 the electron density along the propagation axis as a function of the longitudinal position during the interaction. The solid, dashed-dotted and dotted lines represent the electron density at $t=0, t=33 T$ and $t=41 T$, respectively. At $t=33 T$, the peak of the electron density appears at $20.2 \lambda$ and the electron density is up to $13 n_{c}$, which is already larger than the critical plasma density $n_{c}^{\prime}$. The electron layer is pushed forward continuously by the longitudinal ponderomotive force and the maximum density is more than $25 n_{c}$ at $t=41 T$. Thus, if the width of the target is smaller than the beam waist of the laser pulse, the central part of the laser pulse will be obstructed by the target totally and most of the abaxial laser energy diffracts from the edges of the narrow target and propagate to the rear side of the target, as shown in Fig. 1. On the other hand, if the width of the target is wider than the beam waist, the whole laser pulse will be reflected.

Fig. 6 shows the distribution of the transversal electromagnetic component $E_{y}$ in the $x-y$ plane at (a) $t=25 T$ and (b) $t=41 T$. All parameters are the same as in Fig. 1, except that the width of the target is $20 \lambda$ here. It is seen from the comparison that for the wide target the pulse is fully reflected by the target, no laser energy propagates around the edges of the target. Because the transverse laser intensity is distributed as a Gaussian profile, the strongest longitudinal ponderomotive force will appear on the propagation axis. Due to this, the laser pulse will bore a hole [12] in the front surface and the target appears as a concave mirror. Consequently, the reflected laser pulse is focused towards the propagation axis.

In the mechanism presented here, the second electrostatic field is formed by the electrons released from the edges of the target. If the density of the plasma layer is larger, there will be 
a larger number of electrons at the edges of the target and, hence, a more intense electrostatic field is generated at the back of the target. However, the energetic ions in the front surface will loose more energy in a plasma layer of higher density during their penetration towards the rear side of the target. We therefore expect that there is an optimum parameter set (density, width) for the target to obtain high ion energies.

\section{CONCLUSIONS}

We have presented a new mechanism to generate energetic ions by the interaction of a femtosecond laser pulse with a thin narrow foil. It is shown that most of the laser energy can propagate to the back side of the target, when the target width is narrower than the incident laser beam waist. As a consequence, two electrostatic fields are formed, a first one at the front surface due to the push of the electrons into the target by the longitudinal ponderomotive force. The second electrostatic field at the rear side of the target is formed by electrons released from the edges of the target. Ions from the front surface of the target are accelerated by these two fields to high energies. The energy gain is found to be about four times larger as compared to a wide target.

\section{ACKNOWLEDGMENT}

The project was partially supported by National Natural Science Foundation of China under Grant No. 10375083 and the State Key Basic Research Special Foundation under Grant No. TG1999075206-2. 
[1] E. Fourkal, J.S. Li, W. Xiong, A. Nahum and C.M. Ma, Phys.Med.Biol. 48, 3977 (2003).

[2] M. Roth, T. E. Cowan, M. H. Key et al., Phys. Rev. Lett 86, 436 (2001).

[3] K. Alder, A. Bohr, T. Huus, B. Mottelson and A. Winther, Rev.Mod.Phys. 28, 432(1956).

[4] A. Zhidkov, A. Sasaki, T. Tajima1 et al., Phys. Rev. E 60, 3273 (1999).

[5] A. Maksimchuk, S. Gu, K. Flippo and D. Umstadter, Phys. Rev. Lett. 84, 4108 (2000).

[6] Y. Murakami, Y. Kitagawa, Y. Sentoku, M. Mori, R. Kodama, K. A. Tanaka, K. Mima and T. Yamanaka, Phys. Plasmas 8, 4138 (2001).

[7] A. Macchi, F Cattani, T V. Liseykina and F Cornolti, Phys.Rev.Lett. 94, 165003 (2005).

[8] M. S.Wei, S. P. D. Mangles, Z. Najmudin et al., Phys.Rev.Lett. 93, 155003 (2004).

[9] S. Busch, M. Schnrer, M. Kalashnikov, H. Schnnagel, H. Stiel, P. V. Nickles, W. Sandner, S. Ter-Avetisyan, V. Karpov and U. Vogt, Appl.Phys.Lett. 82, 3354 (2003).

[10] H. Habara, R. Kodama, Y. Sentoku, N. Izumi, Y. Kitagawa, K. A. Tanaka, K. Mima and T. Yamanaka, Phys.Rev.E 69, 036407 (2004).

[11] A.Pukhov, Phys.Rev.Lett. 86, 3562 (2001).

[12] S.C.Wilks, W. L. Kruer, M. Tabak and A. B. Langdon, Phys.Rev.Lett. 69, 1383 (1992).

[13] V. Yu. Bychenkov, V. N. Novikov, D. Batani, V. T. Tikhonchuk and S. G. Bochkarev, Phys.Plasmas 11, 3242 (2004).

[14] T. Zh. Esirkepov, S. V. Bulanov, K. Nishihara et al., Phys.Rev.Lett. 89, 175003 (2002).

[15] J. Denavit, Phys. Rev. Lett. 69, 3052 (1992).

[16] P. McKenna, K. W. D. Ledingham, J. M. Yang et al., Phys.Rev.E 70, 036405 (2004).

[17] S. P. Hatchett, Curtis G. Brown, Thomas E. Cowan et al., Phys.Plasmas 7, 2076 (2000).

[18] R.A.Snavely, M. H. Key, S. P. Hatchett et al., Phys. Rev. Lett 85, 2945 (2000)

[19] E. L. Clark, K. Krushelnick, M. Zepf, F. N. Beg, M. Tatarakis, A. Machacek, M. I. K. Santala, I. Watts, P. A. Norreys and A. E. Dangor, Phys. Rev. Lett. 85, 1654 (2000).

[20] K. Nemoto, A. Maksimchuk, S. Banerjee, K. Flippo, G. Mourou, D. Umstadter and V. Yu. Bychenkov, Appl.Phys.Lett. 78, 595 (2003).

[21] G. S. Sarkisov, V. Yu. Bychenkov, V. N. Novikov and V. T. Tikhonchuk, Phys. Rev. E 59, $7042(1999)$.

[22] K. Krushelnick, E. L. Clark, Z. Najmudin, M. Salvati, M. I. K. Santala, M. Tatarakis and A. 
E. Dangor, Phys. Rev. Lett. 83, 737 (1999).

[23] S. Karsch, S. Dsterer, H. Schwoerer, F. Ewald, D. Habs, M. Hegelich, G. Pretzler, A. Pukhov, K. Witte and R. Sauerbrey, Phys.Rev.Lett. 91, 015001 (2003).

[24] D. Umstadter, Phys.Plasmas 8, 1774 (2001).

[25] A. Zhidkov and M. Uesaka, Phys.Rev.Lett. 89, 215002 (2002).

[26] Han Xu, W. Yu, P. Lu, V. K. Senecha, F. He, B. Shen, L. Qian, R. Li and Z. Xu, Phys.Plasmas 12, 013105 (2005). 
FIG. 1: (color online) The distribution of $E_{y}$ at (a) $\mathrm{t}=33 \mathrm{~T}$ and (b) $\mathrm{t}=41 \mathrm{~T}$ during the interaction of the laser pulse and a narrow target. Here the laser intensity is $1.38 \times 10^{20} \mathrm{~W} / \mathrm{cm}^{2}$, and the radius for the beam waist is $5 \lambda$, where the wave length $\lambda=1 \mu \mathrm{m}$. The width and thickness for the narrow target are $3 \lambda$ and $1 \lambda$, respectively. The target density is $5 n_{c}$, where $n_{c}$ is the critical density.

FIG. 2: The electron (left column) and ion (right column) distributions for the narrow target irradiated by the femtosecond laser pulse. (a),(c) $t=41 \mathrm{~T}$ and (b), (d)t=58T.

FIG. 3: (color online) The longitudinal electrostatic fields at three different time steps, namely $\mathrm{t}=25 \mathrm{~T}$ (dotted line), 37T(solid line), and 49T(dashed-dotted line). The insert shows an enlargement of the region $18 \lambda \leq x \leq 22 \lambda$.

FIG. 4: The ion energy distribution at $\mathrm{t}=58 \mathrm{~T}$ for the (a)wide $(20 \lambda)$ and (b) narrow $(3 \lambda)$ target.

FIG. 5: (color online) The electron density distribution along the propagation axis. The solid, dashed-dotted and dotted lines represent the distributions at $t=0, t=33 T$, and $t=41 T$, respectively. The parameters are same as those in Fig.1.

FIG. 6: (color online) The distribution of $E_{y}$ at (a) $\mathrm{t}=25 \mathrm{~T}$ and (b) $\mathrm{t}=41 \mathrm{~T}$ during the interaction of the laser pulse with a wide $(20 \lambda)$ target. Other parameters are same as those in Fig.1. 


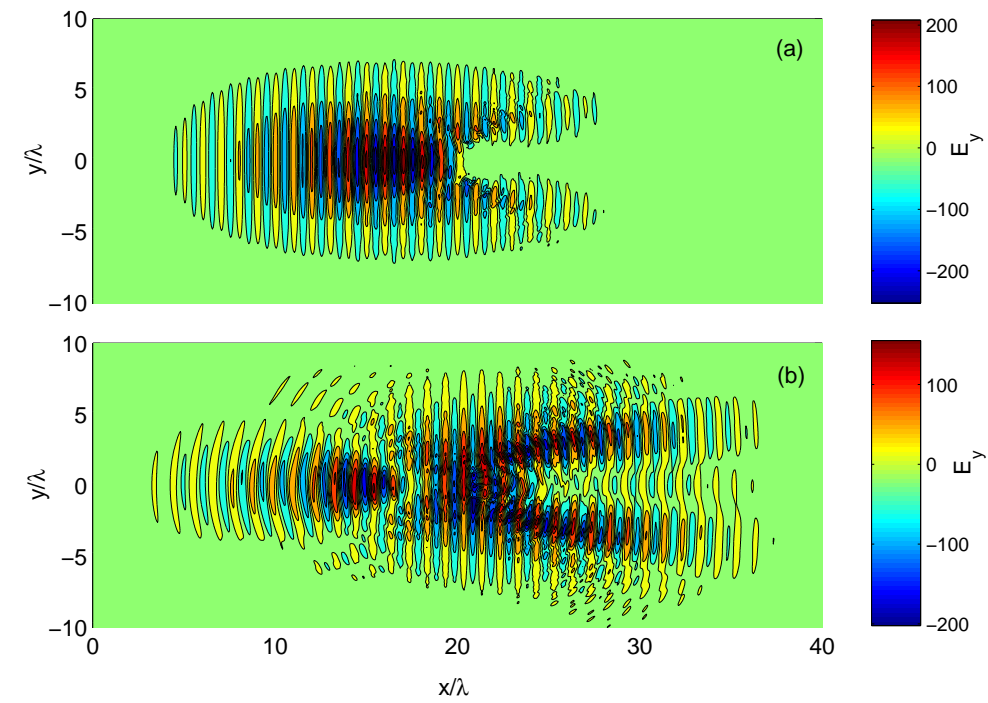

Fig. 1, He et al. 

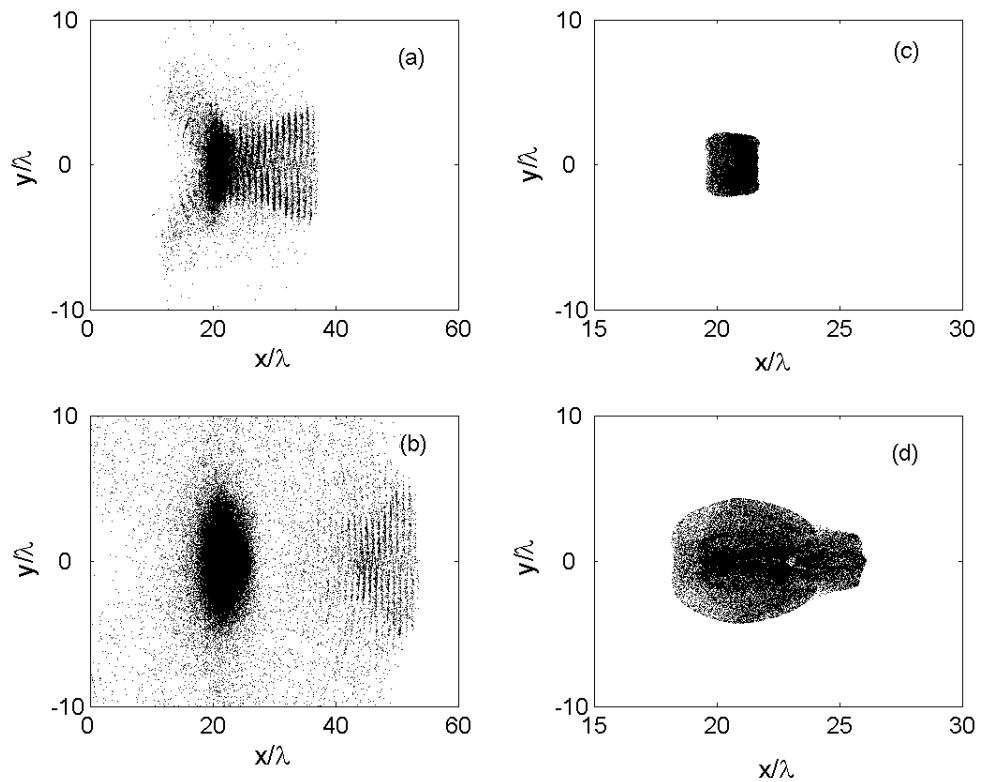

Fig. 2, He et al. 


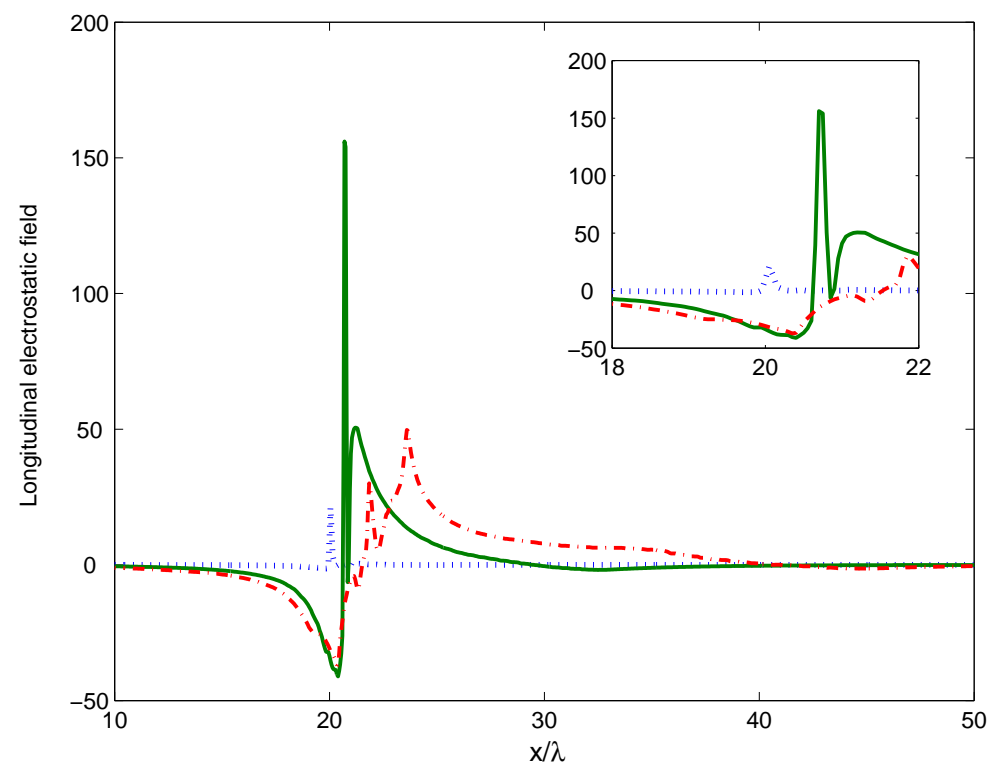

Fig. 3, He et al. 

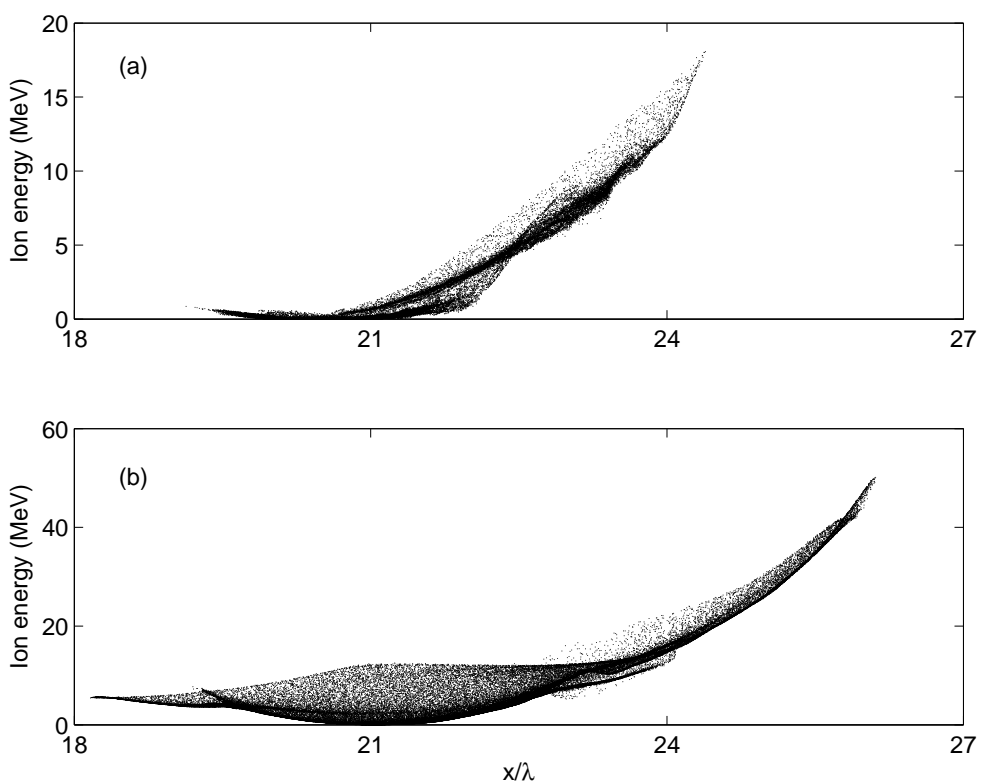

Fig. 4, He et al. 


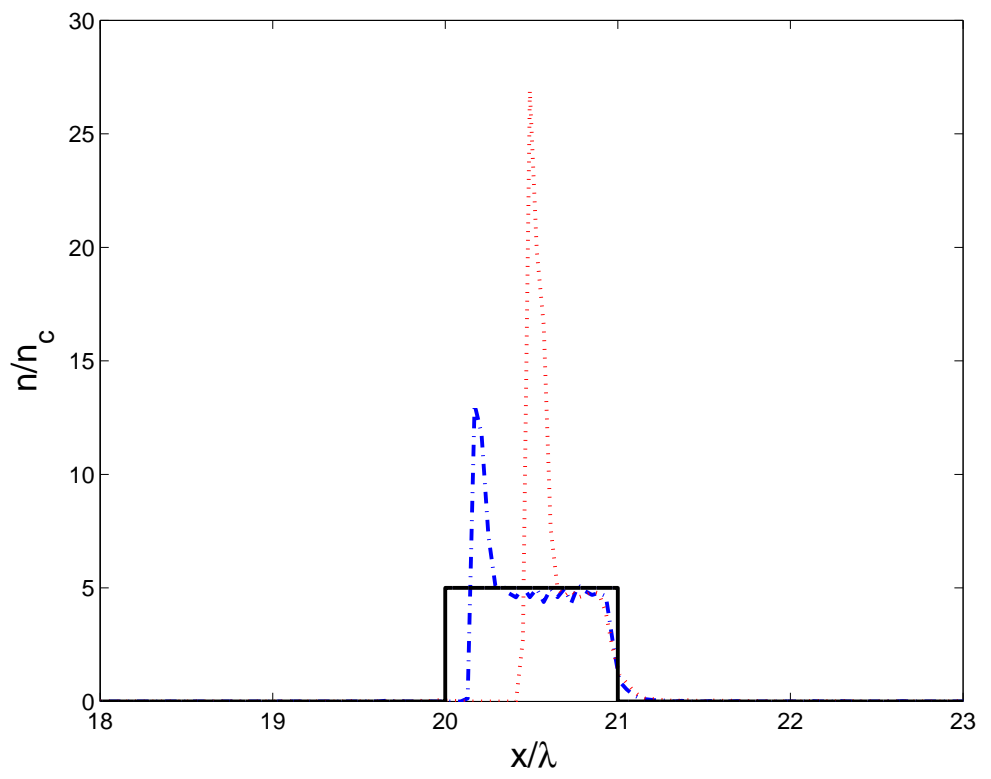

Fig. 5, He et al. 


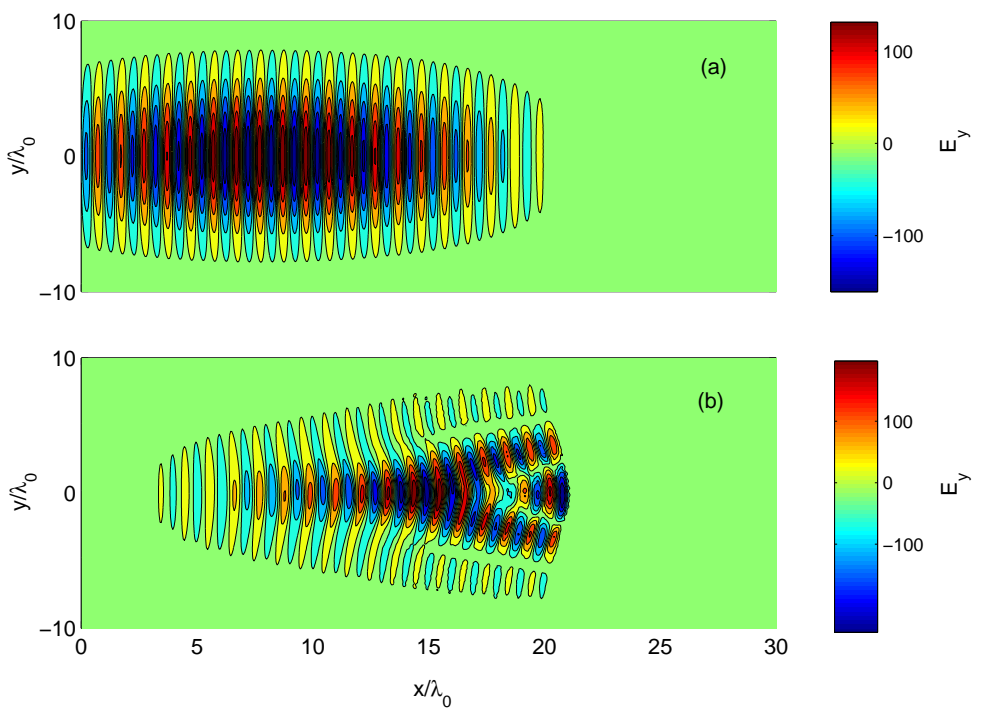

Fig. 6, He et al. 